\title{
Hyaluronan synthase mediates dye translocation across liposomal membranes
}

Andria P Medina', Jialing Lin ${ }^{1,2}$ and Paul H Weigel ${ }^{1,2,3^{*}}$

\begin{abstract}
Background: Hyaluronan (HA) is made at the plasma membrane and secreted into the extracellular medium or matrix by phospolipid-dependent hyaluronan synthase (HAS), which is active as a monomer. Since the mechanism by which HA is translocated across membranes is still unresolved, we assessed the presence of an intraprotein pore within HAS by adding purified Streptococcus equisimilis HAS (SeHAS) to liposomes preloaded with the fluorophore Cascade Blue (CB).

Results: CB translocation (efflux) was not observed with mock-purified material from empty vector control E. coli membranes, but was induced by SeHAS, purified from membranes, in a time- and dose-dependent manner. CB efflux was eliminated or greatly reduced when purified SeHAS was first treated under conditions that inhibit enzyme activity: heating, oxidization or cysteine modification with N-ethylmaleimide. Reduced CB efflux also occurred with SeHAS K48E or K48F mutants, in which alteration of K48 within membrane domain 2 causes decreased activity and HA product size. The above results used liposomes containing bovine cardiolipin (BCL). An earlier study testing many synthetic lipids found that the best activating lipid for SeHAS is tetraoleoyl cardiolipin (TO-CL) and that, in contrast, tetramyristoyl cardiolipin (TM-CL) is an inactivating lipid (Weigel et al, J. Biol. Chem. 281, 36542, 2006). Consistent with the effects of these CL species on SeHAS activity, CB efflux was more than 2fold greater in liposomes made with TO-CL compared to TM-CL.

Conclusions: The results indicate the presence of an intraprotein pore in HAS and support a model in which HA is translocated to the exterior by HAS itself.
\end{abstract}

\section{Background}

Hyaluronic acid (hyaluronan; HA) is a linear unbranched polysaccharide composed of repeating disaccharide units of $\mathrm{N}$-acetyl-glucosamine and $\mathrm{D}$-glucuronic acid. In vertebrates and streptococcal species, HA is synthesized by Class I HA synthases (HAS), lipid-dependent integral membrane proteins with eight membrane domains (MDs) in eukaryotes or six MDs in species such as S. pyogenes and S. equisimilis [1,2]. Mammals express three HAS isozymes encoded by distinct genes designated HAS1, HAS2 and HAS3. HA is an essential glycosaminoglycan in vertebrate extracellular matrices [3], where it helps maintain the physical structure and integrity of tissues, in particular cartilage. HA is also a

\footnotetext{
* Correspondence: paul-weigel@ouhsc.edu

'Department of Biochemistry \& Molecular Biology, The University of Oklahoma Health Sciences Center (940 S. L. Young Blvd), Oklahoma City, OK 73104, USA

Full list of author information is available at the end of the article
}

major constituent of skin, vitreous humor in the eye, synovial fluid in joints, and the cumulus cell matrix that surrounds oocytes prior to ovulation. In tissue matrices or vitreous, HA ranges in molecular mass up to $10 \mathrm{MDa}$ (25, 000 disaccharide units) and occupies a very large volume in physiological fluids. In addition to its physical functions, it is now clear that HA has multiple different size-dependent functions and is capable of stimulating intracellular signal transduction to alter gene expression and cell behavior in angiogenesis, inflammatory diseases, tumorigenesis and metastasis [4-8].

Two mechanisms have been suggested for how HA is transferred across the cell membrane to the exterior. The first [9] is that HAS itself, which is active as a monomer in complex with multiple lipids molecules [10], mediates HA translocation via a pore within the multiple MDs of the protein. The second is that a growing $\mathrm{HA}$ chain in the cytoplasm binds to an $\mathrm{ABC}$ transporter, which then transfers the chain across the 
membrane $[11,12]$. This suggestion arose when mutation of a gene near the $S$. pyogenes has operon reduced HA synthesis and secretion by about 65\%, although SpHAS activity in lysed cells was unaffected. The mutated gene had sequence similarity to bacterial $\mathrm{ABC}$ transporter genes. In eukaryotic cells, the current proposal is that both the multiple drug resistance protein-5 (MRP5; $\mathrm{ABCC} 5$ ) and the cystic fibrosis conductance regulator are $A B C$ transporters that would bind and translocate intracellular HA to the exterior in an ATP-dependent manner $[13,14]$.

The HAS Pore model is based on three aspects of HAS structure and function that are very novel for a glycosyltransferase: the lipid-dependence, the large number of MDs, and the processive mechanism of HA synthesis. Very few glycosyltransferases are multiple membrane spanning proteins and dependent on lipid for activity. HA.HAS complexes remain associated for 2-4 hr until the completed MDa HA chains are released [15]. Additional support for the Pore model is that insertion of a HAS gene into cells that do not otherwise make HA can confer the ability to synthesize and secrete HA in prokaryotes [16,17] or eukaryotes such as drosophila [18]. These results are readily explained by the ability of HAS alone to both assemble and translocate HA. The ABC transporter model explains these latter results by proposing that the transporters in cells that do not make HA are promiscuous enough to transport not only their normal substrates, but also the large HA polysaccharide made when a HAS gene is introduced. A further distinction is that in contrast to the $\mathrm{ABC}$ transporter model, the HAS Pore model with coupled HA synthesis and translocation does not require ATP.

In this study, we tested a central prediction of the Pore model, that HAS contains an intraprotein pore, by assessing the ability of purified SeHAS to mediate efflux (i.e. translocation) of the small fluorescent dye Cascade Blue (CB, $538.4 \mathrm{Da})$ from the inside of pre-loaded liposomes to the external medium. The results indicate that SeHAS can mediate CB efflux when added to liposomes and that this pore-like activity is eliminated or impaired by a range of treatments or conditions that inhibit the synthase activity of SeHAS.

\section{Results}

\section{CB Efflux occurs after SeHAS addition to liposomes}

When $\mathrm{CB}$ dye is released from liposomes and bound by anti-CB antibody $(\mathrm{Ab})$ in the external phase (Figure 1), its fluorescence (FL) is quenched. Luminal CB does not readily leak through the liposomoal membrane, and hence, is protected from the quenching. However, $C B$ release can occur in several ways, including detergent mediated lysis, loss of liposome integrity (e.g. due to

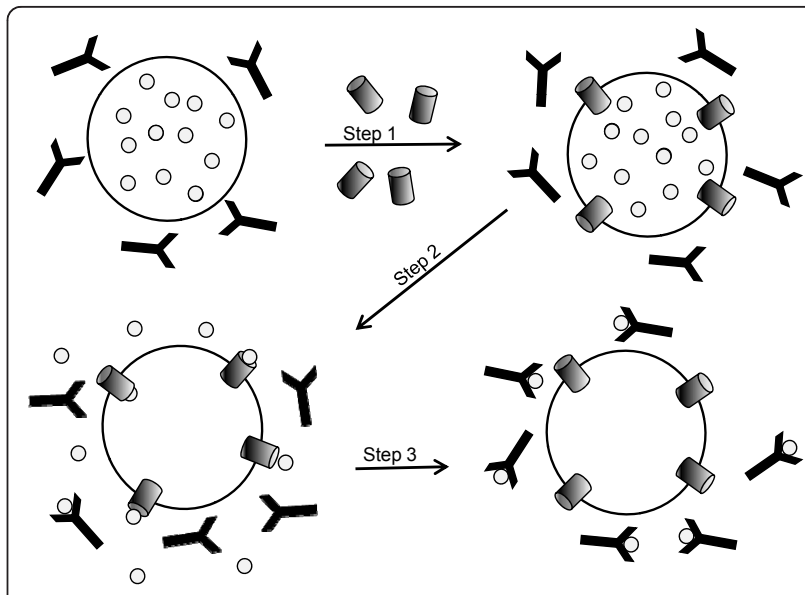

Figure 1 Experimental model for monitoring HAS-mediated FL quenching in liposomes. Step 1 in the scheme depicts the addition of purified SeHAS (cylinders) to extrusion liposomes (large circles) containing free CB (small circles). In step 2, HAS molecules insert into the liposome bilayer, allowing the small CB dye to translocate (efflux) through the HAS pores to the exterior if an intraprotein pore is present. In experiments with continuous FL monitoring in the presence of external anti-CB Ab (black Y), CB that effluxes from the lumen is bound by $A b$ resulting in $F L$ quenching (step 3).

aging-induced lipid oxidation and degradation), or by passage through pores formed by insertion of proteins into liposomal membranes [19,20]. For these experiments, SeHAS was purified using a protocol with reduced $n$-dodecyl- $\beta$-D-maltoside (DDM) and glycerol concentrations to minimize carryover of any detergent. Prior to protein addition or in the absence of addition, a stable FL baseline was observed, indicating that no $\mathrm{CB}$ dye is released spontaneously from liposomes at $30^{\circ} \mathrm{C}$. After SeHAS addition, there was a time-dependent decrease in FL (an increase in quenching) indicating an ongoing efflux of CB (Figure 2A). In contrast, there was no quenching when the same buffer without SeHAS or when mock-purification material from empty-vector membranes was added. The latter control indicates that $\mathrm{CB}$ efflux from liposomes was not caused by residual DDM or other membrane contaminants. Essentially all of the added SeHAS was incorporated into the liposomes under these conditions (Figure 2B). Additionally, SeHAS did not cause dye release and quenching when added to liposomes containing a larger (3 kDa) dextranCB conjugate (not shown).

\section{CB efflux from liposomes is dependent on SeHAS concentration}

If $\mathrm{CB}$ efflux is mediated by SeHAS insertion into liposomes, this effect should be dose dependent. When this was assessed using different amounts of purified SeHAS, the resulting $\mathrm{CB}$ efflux was dependent on SeHAS 

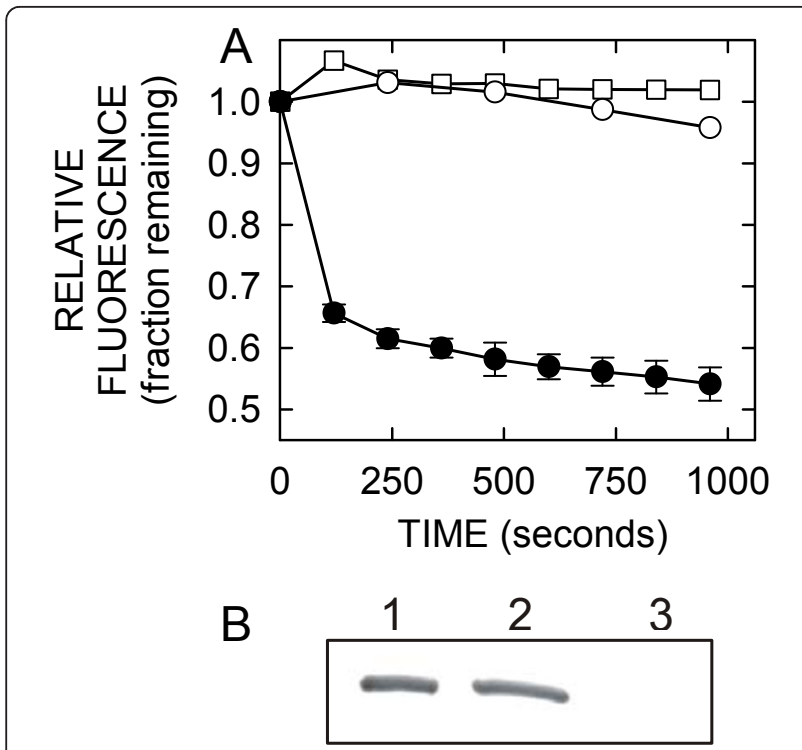

Figure 2 Incorporation of SeHAS into extrusion liposomes induces $\mathrm{CB}$ quenching. $\mathrm{A}$. Extrusion liposomes loaded with $\mathrm{CB}$ were prepared, purified and stored at $4^{\circ} \mathrm{C}$ under argon as described in Methods. Liposomes were suspended in $0.25 \mathrm{ml} 0.1 \mathrm{M} \mathrm{NaCl}, 51$ mM sodium phosphate, $3.8 \mathrm{mM}$ Na citrate, $\mathrm{pH} 7.4$ ( 50 $\mu \mathrm{M} \mathrm{PL}$ ) with $10 \mu \mathrm{g} / \mathrm{ml}$ anti-CB Ab and equilibrated at $30^{\circ} \mathrm{C}$ in cuvettes in the fluorometer. FL was monitored at $433 \mathrm{~nm}$ for $5 \mathrm{~min}$ to obtain a stable base-line (time-zero), and then SeHAS ( $15 \mu$; purified with no DDM or glycerol in the elution buffer) was added to $200 \mathrm{nM}$ (black circles; mean $\pm S E, n=3$ ) or the same volume of mock-purified empty-vector membranes (white circles) or elution buffer (white squares) was added. Samples were mixed well and FL monitoring was continued. Decreasing FL (ongoing quenching) was observed after SeHAS addition, but not after addition of elution buffer or mock-purified material from empty-vector membranes. B. Purified SeHAS was added to extrusion liposomes as in A and after 30 min the suspension was centrifuged, the supernatant was saved, and the pellet was resuspended and centrifuged. The final pellet (lane 2) was resuspended to the same volume as the original supernatant (lane 3), trichloroacetic acid was added (to 10\%; w/v) to both samples and precipitated protein was centrifuged, redissolved in Laemmli buffer and analyzed by SDS-PAGE and Western blotting with anti-SeHAS Ab. Lane 1 is a sample of purified SeHAS precipitated and redissolved in parallel as a control.

concentration (Figure 3). After adding SeHAS to 50, 100 , or $200 \mathrm{nM}$, the quenching after $30 \mathrm{~min}$ was $3 \%, 6 \%$ and $14 \%$, respectively. Thus, essentially a linear response in CB efflux was observed with increasing SeHAS concentration, consistent with the interpretation that $\mathrm{CB}$ efflux is mediated by, and dependent on, SeHAS.

\section{SeHAS-mediated CB efflux is greater from liposomes made with an activating compared to an inactivating lipid}

If $\mathrm{CB}$ efflux is mediated by native active SeHAS, then this response should be increased by conditions that stimulate enzyme activity and decreased by treatments

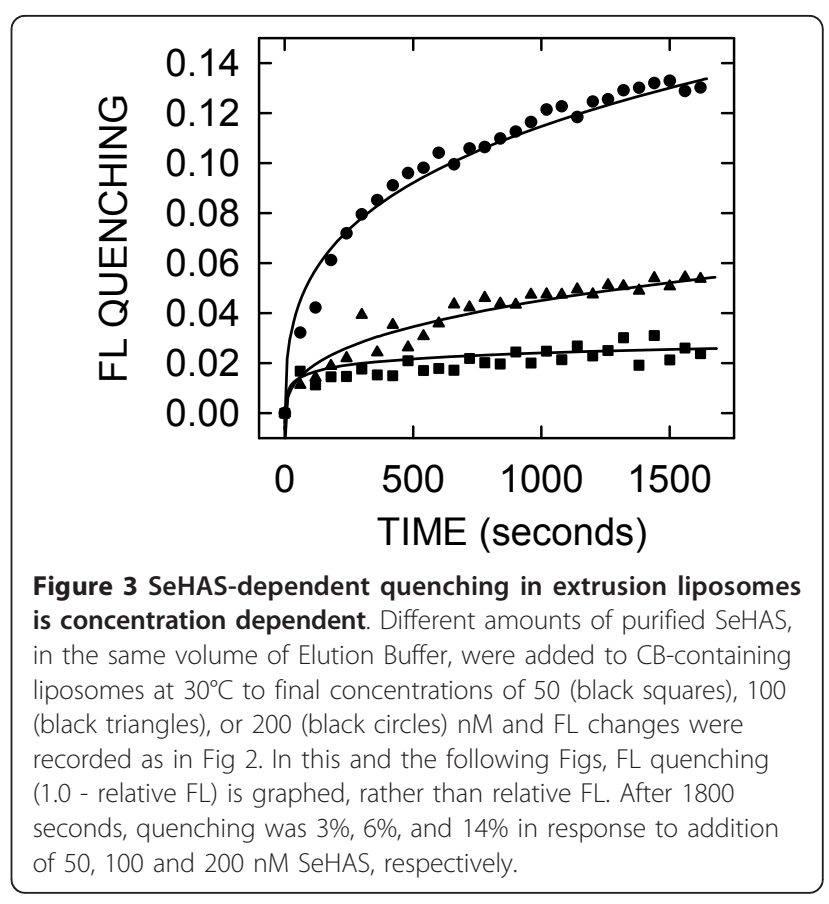

that inhibit or inactivate enzyme activity. SeHAS and other Class I HAS enzymes are lipid-dependent $[1,9,21]$. For SeHAS, we found that tetraoleoyl cardiolipin (TO$\mathrm{CL}$ ), containing C18:1 oleoyl groups (with one double bond), is the best activating phospholipid, whereas tetramyrstoyl cardiolipin (TM-CL) with saturated C14:0 myristyl groups actually inhibits SeHAS activity [22]. Earlier radiation inactivation studies found that active native and recombinant streptococcal synthases are a complex of one HAS monomer and $\sim 16$ cardiolipin molecules [10]. The liposomes used here were made with $5 \%$ bovine cardiolipin (BCL), which contains several CL species including TO-CL and is an activating lipid for SeHAS [21].

To determine if CB efflux mediated by SeHAS is influenced by the CL species present, we tested liposomes made with an $E$. coli-based lipid composition of phosphatidyglycerol (PG) and phosphatidyethanolamine (PE) and either $5 \%$ TO-CL or TM-CL, in place of BCL. The maximal quenching induced by SeHAS addition to liposomes made with TO-CL was more than 2-fold greater than in liposomes made with TM-CL (Figure 4). Interestingly, quenching using TM-CL liposomes was $~ 20 \%$ less compared to the standard liposomes made with $\mathrm{BCL}$, but quenching in liposomes made with TO-CL was $\sim 50 \%$ greater.

Fluorescence quenching does not occur after addition of heat-inactivated, oxidized or Cys-modified SeHAS

To determine if the observed $\mathrm{CB}$ efflux required active native SeHAS, we inactivated the enzyme by heating for 


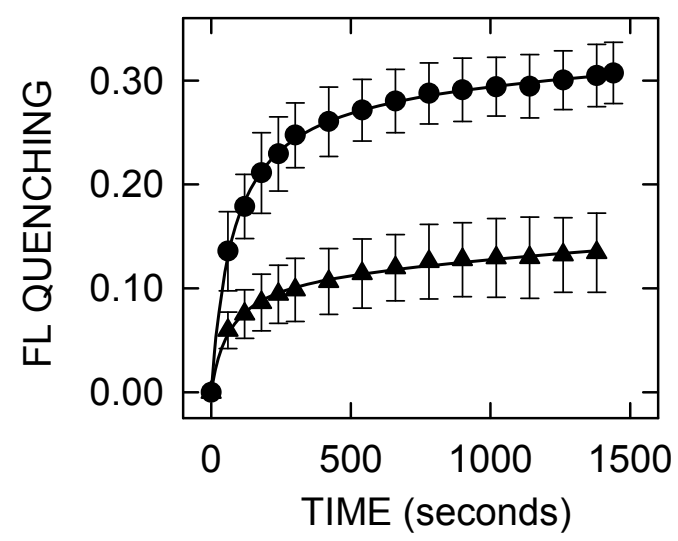

Figure 4 SeHAS mediates greater $C B$ release from extrusion liposomes containing activating $\mathrm{TO}-\mathrm{CL}$ compared to inhibitory TM-CL. Purified SeHAS was added to liposomes containing (mol\%) 70\% PE, 25\% PG and either 5\% TO-CL (black circles) or TM-CL (black triangles). Subsequent release of $\mathrm{CB}$ and $\mathrm{FL}$ quenching by anti-CB $\mathrm{Ab}$ was monitored as described in Fig 2. Values are the mean \pm SE $(n=$ 3). Note that the FL quenching scale is greater than in other Figs.

$5 \mathrm{~min}$ at $47^{\circ} \mathrm{C}$ [21]. There was no quenching of $\mathrm{CB} F L$ when this inactive SeHAS was added to liposomes (Figure $5 \mathrm{~A}$ ), indicating that native enzyme is required. It has been known for $>50$ years that HA synthesis does not occur if the enzyme is oxidized; thus, a reducing agent is required to maintain enzyme activity [23]. To assess the ability of oxidized SeHAS to mediate CB efflux, we omitted DTT during the standard elution step, which resulted in inactive enzyme (not shown) that was unable to mediate CB efflux from liposomes (Figure 5B). Reducing the oxidized SeHAS by adding back DTT recovered both enzymatic activity and the ability to mediate $\mathrm{CB}$ efflux. Although Cys residues in SeHAS or SpHAS are not required for enzyme activity, their modification by NEM or other sulfhydryl reagents drastically reduces activity [24-26]. Adding SeHAS pretreated with NEM to liposomes resulted in $\sim 75 \%$ less quenching compared to unmodified enzyme, whereas addition of NEM alone did not quench CB FL (Figure 5C). Thus, three different treatments that inhibit HAS activity also decreased the ability of SeHAS to mediate CB efflux.

\section{CB efflux from liposomes is reduced in SeHAS MD mutants}

We reported previously that two charged residues within MD2 (Lys ${ }^{48}$ ) and MD4 $\left(\mathrm{Glu}^{328}\right)$ appear to act together (e.g. as an ion pair) to stabilize the protein and allow synthesis of very large HA [27]. These two MD residues are within the proposed intraprotein pore of HAS. The $V_{\text {max }}$ of SeHAS(K48E), a charge-switch mutant, is reduced by $93 \%$ and it makes HA that is $81 \%$ smaller compared to WT SeHAS. CB quenching after addition of SeHAS(K48E) to liposomes was reduced by
$66 \%$ compared to WT (Figure 6A). We also tested a $\mathrm{K} 48 \mathrm{~F}$ variant because the replacement residue has a bulky side group and this mutant makes HA that is $\sim 77 \%$ smaller than WT. FL quenching was reduced by $\sim 45 \%$ compared to addition of WT SeHAS (Figure 6B). These results indicate that $\mathrm{CB}$ efflux mediated by SeHAS is inhibited by mutations in the putative pore region that decrease both enzyme activity and HA product size.

\section{Discussion}

The present results demonstrate that purified HAS reconstituted into liposomes mediates luminal $\mathrm{CB}$ efflux in a manner consistent with the presence of a HAS pore. HAS-mediated dye efflux was time-dependent and HAS concentration-dependent, and efflux did not occur or was greatly impaired if the enzyme was first inactivated by heat treatment or $\mathrm{Cys}-\mathrm{SH}$ group modification by either oxidation or reaction with NEM. These results indicate that active native enzyme is required for efflux. HAS enzyme function is greatly affected by whether sufficient activating phospholipid is present. We previously found that purified SeHAS retains enough tightly bound endogenous lipid to retain low activity (and not be denatured), but the enzyme can be activated $\geq 10$-fold by addition of TO-CL, containing C18:1 oleic acid chains; the best activating lipid identified so far [22]. In contrast, TM-CL, containing C14:0 saturated fatty acids, does not activate SeHAS. The finding that HASmediated dye efflux was greater in liposomes with TOCL compared to BCL and greater with BCL versus TM$\mathrm{CL}$ is consistent with the presence of a pore whose size is dependent on the associated CL species.

An intraprotein pore in HAS-CL complexes may be smaller or distorted (thus slowing the rate of efflux) in the presence of the "bad" TM-CL species. Unlike other phosholipids, CL (also called diphosphatidylglycerol) has a "double" head group and four fatty acyl chains. Consequently, whereas other lipids occupy roughly cylindrical volumes, CL is shaped more like a cone with the narrower head group at the top. This structure imparts a natural tendency for CL to create or to localize to areas of negative (i.e. concave) membrane curvature [28]. We conclude that HAS could be activated by TO-CL, which favors negative curvature in the inner leaflet, because it would decrease lateral pressure on the protein and thus open up the cytoplasmic active sites and intraHAS pore [29]. In contrast, HAS inhibition by TM-CL, whose shorter unkinked acyl chains favor a more cylindrical shape and more positive membrane curvature, could occur because it increases lateral pressure on the protein and would narrow the pore.

Since cloning and characterizing the first HAS [30], we have been interested in understanding how HAS 


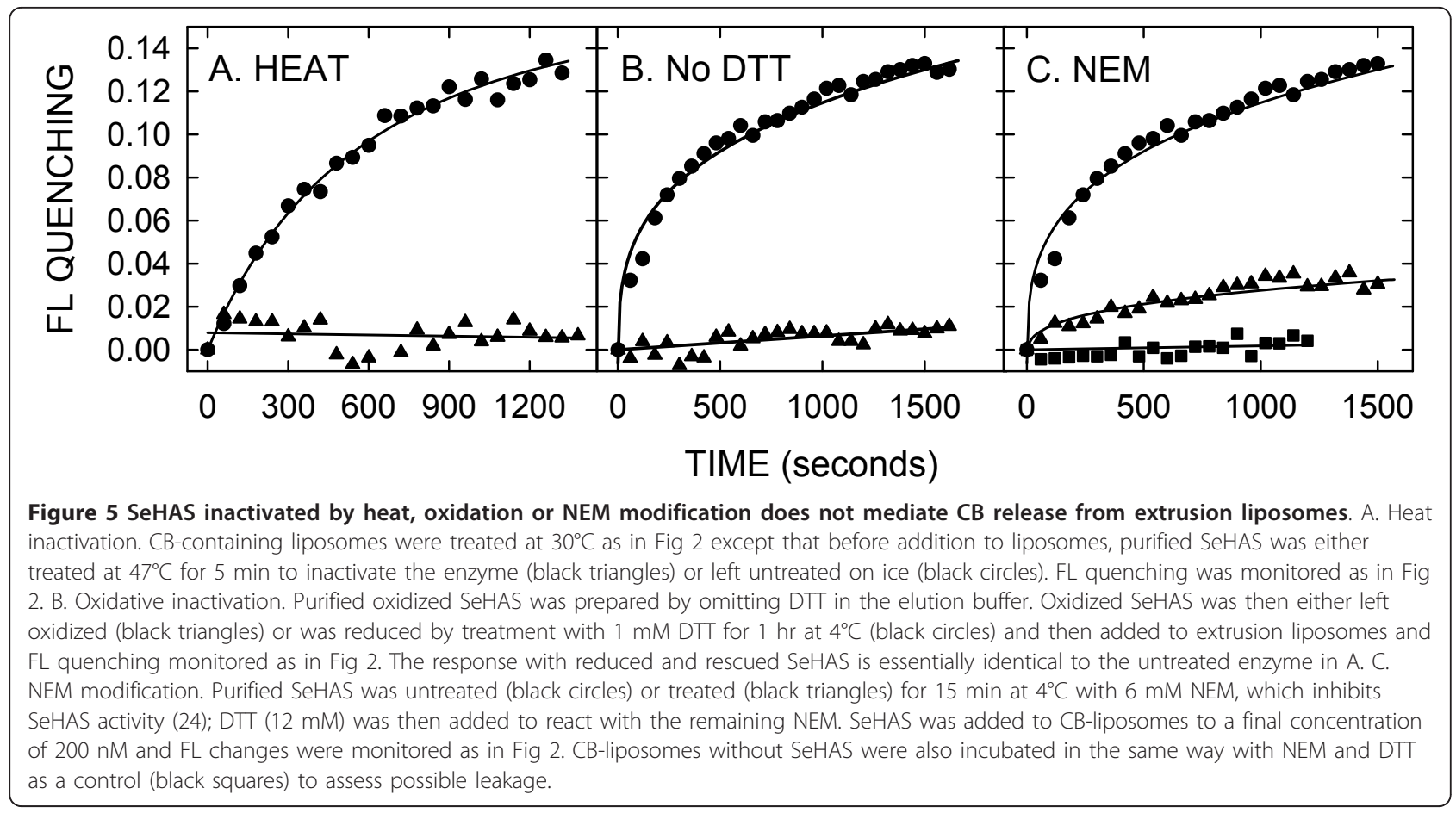

regulates the size of HA chains it assembles and how HA.HAS complexes can stay together for hours without dissociating during continuous polymerization of a growing chain at the reducing end (unlike the vast majority of glycosyltransferases that act at the nonreducing end). The characteristics of the HAS protein itself and how it functions led us to propose the Pore model for HA translocation through and by a HAS-lipid complex $[1,9,31]$. For example, the lipid-dependence and 6-8 MDs of membrane-bound HAS enzymes are characteristics common to pore forming proteins [19,32-35]. Additionally, the substrate binding sites are on the cytoplasmic side of the protein close to the cytoplasmmembrane interface $[2,26]$ and the enzyme is highly processive $[15,36]$.

Processive biosynthesis requires that after each catalytic cycle the HA-UDP product must transiently dissociate, without being released to diffusion, move relative to the enzyme active sites, and then rebind to HAS for the next cycle of sugar additions. As with other polymerases, the binding interaction between enzyme and polymer substrate cannot be of such high affinity that the rate of release, and thus rebinding of polymer, is too slow. Many RNA and DNA enzymes (e.g. polymerases and
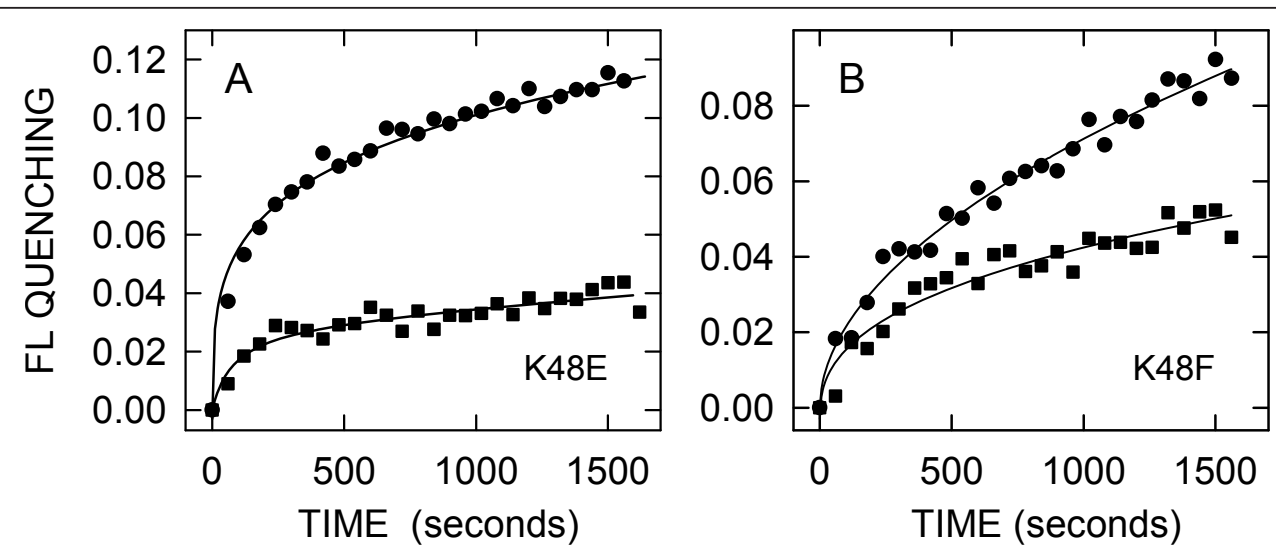

Figure 6 K48E and K48F mutants mediate less CB release from liposomes than WT SeHAS. Purified WT (black circles) and K48E (Panel A, black squares) or K48F (Panel B, black squares) SeHAS were added to $\mathrm{CB}$-containing liposomes and FL quenching was monitored at $30^{\circ} \mathrm{C}$ for the indicated times as in Fig. 2. 
topoisomerases) have evolved solutions to this dilemma by creating topological or spatial constraints so that enzyme and product cannot dissociate. Molecular tethering strategies include formation of either multiprotein complexes that surround and encase the polymeric substrate or an intra-enzyme channel or pore to achieve a similar entrapment of the polymer.

This processivity of HA.HAS complexes is most consistent with the Pore model, which provides a topological mechanism to prevent dissociation during HA elongation. Since the 6-8 MDs of HAS enzymes are associated with multiple lipid molecules, it seems most likely that they utilize an intraHAS pore or deep cleft within the membrane that simultaneously serves to topologically constrain and also to provide a translocation mechanism to move the growing chain to the cell exterior. When dissociation does occur, HA release appears to terminate further elongation, perhaps due to loss of UDP at the reducing end - a chain terminating event. Even high affinity interactions (e.g. between receptors and ligands) typically have $n M K_{d}$ values and measurable off-rates. The extremely slow, essentially immeasurable, off-rate for HA.HAS complex dissociation is inconsistent with enzyme reactions that use soluble substrates, which typically have $K_{m}$ values in the $\mu \mathrm{M}$ range, as proposed in the $\mathrm{ABC}$ transport model.

We previously identified Lys ${ }^{48}$ and Glu ${ }^{327}$ in MD2 and MD4, respectively, as important residues for the ability of SeHAS to produce large MDa HA [27]; the K48E and K48F mutants make much smaller HA than WT. The interpretation that these two residues are within the proposed HAS pore and may interact with each other or with the growing HA chain during translocation is supported by the present findings that $\mathrm{CB}$ efflux mediated by both proteins is decreased compared to WT.

Since the present evidence supports the presence of an intraHAS pore through which HA could be translocated across membranes, it is reasonable to consider alternative interpretations that could account for reduced HA synthesis and secretion by inhibition of $\mathrm{ABC}$ transporters, such as MRP5. Two other explanations for decreased HA synthesis and secretion after inhibition or knockdown of MRP5 and other ABC transporters are related to the ability of these proteins to transport nucleotides (e.g. cGMP, cAMP) as well as nucleotidebased drugs such as 5'-Fluoro-UMP, a metabolite of the anticancer drug 5'-Fluorouracil [37]. The first possibility is that HAS inhibition could be caused by perturbation of the normal cellular metabolism of uracil-containing nucleotides, resulting in altered UDP-sugar concentrations or ability of precursor pathways to sustain the metabolic flux needed to make large amounts of HA. In eukaryotes, UDP-sugars are synthesized in the cytoplasm and levels are controlled by several mechanisms, including the Golgi anti-port system in which a UDP-sugar is transported in, while UMP is transported out. UMP deficiency causes the metabolic disorder orotic aciduria, but excess UMP (which leads to excess UDP) would also have a detrimental effect on HA synthesis. UDP is a potent substrate inhibitor of all Class I HASs [1] and, in fact, can be used to quench HA synthase activity [15]. Thus, deficiency or inhibition of MRP5 could lead to accumulation of cytoplasmic UDP and inhibition of HAS, an indirect result of MRP5 function unrelated to HA translocation. Both of the above possible indirect effects on HAS activity would be reversed by lysing cells (i.e. dilution and mass action effects) and performing assays with UDP-sugars added in vitro. Although these and other alternate explanations remain to be tested, Thomas and Brown recently found that $\mathrm{ABC}$ transporters are not involved in HA export by cancer cells [38].

\section{Conclusions}

We conclude that HAS contains an intraprotein pore capable of allowing small molecules such as Cascade Blue (538.4 Da) to pass across liposomal membranes. Such a pore within HAS provides both an explanation for the extreme processive behaviour of HAS.HA complexes, which remain associated for hours during biosynthesis of MDa HA chains, and a mechanism for the translocation of HA across cell membranes to the cell exterior that is consistent with the enzyme's structure and lipid dependence.

\section{Methods}

\section{Materials and Buffers}

Media components were from Difco (Fisher Scientific). Plasmid pKK223-3 was from GE Healthcare and E. coli, SURETM cells were from Stratagene (La Jolla, CA). $\mathrm{Ni}^{2}$ ${ }^{+}$-nitrilotriacetic acid (Ni-NTA) resin was from Qiagen Inc (Hilden, Germany). CB (8-methoxypyrene-1, 3, 6-trisulfonic acid, trisodium salt, 538.4 Da) and anti-CB quenching antibody $\mathrm{Ab}$ were from Invitrogen-Molecular Probes. Synthetic TO-CL and TM-CL were from Avanti Polar Lipids (Alabaster, Alabama). BCL, E. coli PE, and chicken egg PG were from either Avanti or SigmaAldrich. Other reagents were from Sigma-Aldrich (St. Louis, MO). Liposome Buffer contains $100 \mathrm{mM} \mathrm{NaCl}$, $51 \mathrm{mM} \mathrm{Na} \mathrm{HPO}_{4}, 3.8 \mathrm{mM}$ citric acid, $\mathrm{pH}$ 7.4. Extraction Buffer contains $10 \mathrm{mM}$ DDM, $50 \mathrm{mM}$ sodium and potassium phosphate, $\mathrm{pH} 7.0,150 \mathrm{mM} \mathrm{NaCl}, 10 \mathrm{mM}$ $\mathrm{MgCl}_{2}, 1.0 \mathrm{mM} \beta$-mercaptoethanol, 20\% glycerol, 0.5 $\mu \mathrm{g} / \mathrm{ml}$ leupeptin, $0.7 \mu \mathrm{g} / \mathrm{ml}$ pepstatin, and $46 \mu \mathrm{g} / \mathrm{ml}$ phenylmethylsulfonyl fluoride. Wash and Elution Buffers contain the same components except that in Wash Buffer glycerol is $10 \%$ and DDM is $1 \mathrm{mM}$, and Elution Buffer does not contain either glycerol or DDM. 


\section{Cell growth and membrane purification}

The HAS open reading frame from $S$. equisimilis, with a C-terminal $\mathrm{His}_{6}$ fusion, was inserted into the pKK223-3 vector and cloned into E. coli SURE-2 cells [17], and cell growth, lysozyme/EDTA treatment, and membrane preparation in the presence of protease inhibitors were performed as described previously $[9,21]$. Final membrane pellets were stored at $-80^{\circ} \mathrm{C}$.

\section{Enzyme extraction and purification}

In order to minimize the residual detergent associated with purified SeHAS to be used with liposomes, the Extraction, Wash and Elution buffers used in previous purification schemes were modified, as noted above, to reduce or eliminate glycerol and DDM. Thawed membrane pellets were solubilized in $10 \mathrm{ml}$ of Extraction Buffer for $2 \mathrm{~h}$ at $4^{\circ} \mathrm{C}$ with gentle mixing in a Micromixer E36 (Taitec), followed by centrifugation at $100,000 \times \mathrm{g}$ for $1 \mathrm{~h}$ at $4^{\circ} \mathrm{C}$ to sediment insoluble components. Imidazole was added to the supernatant to a final concentration of $30 \mathrm{mM}$ to minimize nonspecific binding of $E$. coli proteins and the extract was incubated for $30 \mathrm{~min}$ at $4^{\circ} \mathrm{C}$ with constant mixing with $\mathrm{Ni}^{2+}$-depleted NTA resin $(0.3$ $\mathrm{ml}$ in a mini-spin column; Bio-Rad) equilibrated with Extraction Buffer without $\mathrm{MgCl}_{2}$. The enzyme extract was removed and then incubated with $0.3 \mathrm{ml} \mathrm{Ni}^{2+}$-NTA resin for $2 \mathrm{~h}$ at $4^{\circ} \mathrm{C}$ with constant mixing. After allowing the resin to pack, unbound proteins were allowed to flow through, the resin was washed with 10 volumes of Wash Buffer to remove contaminants, and bound SeHAS was recovered with $0.3 \mathrm{ml}$ Elution Buffer. SeHAS protein was determined with the Coomassie protein assay reagent (Pierce) using bovine serum albumin as the standard, and purity was assessed by SDS-PAGE; preparations were typically $>98 \%$ pure based on densitometric analysis of Coomassie-stained gels.

\section{Extrusion liposomes}

Liposomes containing CB were prepared as described by Szoka et al $[39,40]$. Since recombinant SeHAS in E. coli membranes has been well characterized and is very active, we prepared liposomes (unless noted otherwise in particular experiments) using a phospholipid mixture based on E. coli membranes: 75\% PG, 20\% PE and 5\% CL (in mole percents). To facilitate binding between liposomes and the C-terminal $\mathrm{His}_{6}$ of SeHAS [40], we also added $1 \%$ of a NTA-Ni ${ }^{2+}$ PG analogue (Avanti): 1 , 2-dioleoyl-sn-glycero-3-[(N-(5-amino-1-carboxypentyl) iminodiacetic acid)-succinyl] (nickel salt). Lipids in chloroform or ethanol were mixed and dried under a partial vacuum for $3 \mathrm{~h}$ at $37^{\circ} \mathrm{C}$ in a $\mathrm{N}_{2}$ atmosphere. To the dried lipid mixture was added $0.55 \mathrm{ml}$ of Liposome Buffer and $30 \mu \mathrm{M} \mathrm{CB}$ [19]. The mixture was incubated at $37^{\circ} \mathrm{C}$ for $30 \mathrm{~min}$, and then resuspended by vortex mixing for $5 \mathrm{~min}$. The lipid mixture was frozen in liquid $\mathrm{N}_{2}$ and thawed in a $37^{\circ} \mathrm{C}$ water bath five times to reduce the content of multilamellar liposomes and to increase liposome luminal volume, and then passed 21 times through a $0.2 \mu \mathrm{m}$ pore size polycarbonate filter at room temperature using a Lipofast extruder (Avestin Inc, Ottawa, Canada) to make unilamellar liposomes [39]. Liposomes were stored at $4^{\circ} \mathrm{C}$ until use, and then external $\mathrm{CB}$ was removed from liposomes (containing internal $C B$ ) by either centrifugation or size exclusion chromatography (SEC) over Sephadex G-25.

\section{Monitoring $\mathrm{CB}$ release from Extrusion liposomes}

CB-loaded liposomes (50 $\mu \mathrm{M}$ phospholipid) were equilibrated in $0.250 \mathrm{ml}$ Liposome Buffer containing 6-10 $\mu \mathrm{g} /$ $\mathrm{ml}$ anti-CB $\mathrm{Ab}$ at $30^{\circ} \mathrm{C}$ in a cuvette in an SLM-8100 spectrofluorometer [19]. Gentle mixing was achieved with a flea magnet and magnetic stirrer. FL intensity was measured (excitation at $405 \mathrm{~nm}$ and emission at $433 \mathrm{~nm}$ ) for at least $5 \mathrm{~min}$ until a steady baseline was achieved. CB release from liposomes, upon addition of SeHAS or other agents, was monitored continuously by measuring $\mathrm{CB} F \mathrm{FL}$ in the presence of external anti-CB $\mathrm{Ab}$ that quenches FL. Purified SeHAS $(0.83 \mu \mathrm{M})$ was then added to the cuvette to a concentration of $50 \mathrm{nM}$, the contents mixed, and continuous FL monitoring resumed $20 \mathrm{~s}$ after SeHAS addition (this time point is designated as $t_{o}$ or $0 \mathrm{sec}$ in Figures 2, 3, 4, 5, and 6). At the end of each kinetic run, Triton-X100 was added to a final concentration of $0.1 \%(\mathrm{v} / \mathrm{v})$ to lyse liposomes, completely release $\mathrm{CB}$, and obtain values for maximum (100\%) quenching by the anti-CB Ab. Relative FL is calculated as $\left[\left(\mathrm{F}_{\mathrm{t}}-\mathrm{F}_{\mathrm{T}}\right) /\left(\mathrm{Ft}_{0}-\mathrm{F}_{\mathrm{T}}\right)\right]$ where: $\mathrm{Ft}_{0}=\mathrm{FL}$ at time-zero with anti-CB Ab present, but before SeHAS addition to liposomes; $F_{t}=F L$ at a given time $t$, and $F_{T}$ is the final FL 5 min after adding Triton-X100. FL quenching (Q) is calculated as: $\mathrm{Q}=\left(\mathrm{Ft}_{0}-\mathrm{F}_{\mathrm{t}}\right) /\left(\mathrm{Ft}_{0}-\mathrm{F}_{\mathrm{T}}\right)$. Thus, at any given time $\mathrm{Q}$ represents the efflux and quenching of $\mathrm{CB}$ as a fraction of the total FL quenching of $\mathrm{CB}$ after Triton $\mathrm{X}-100$ addition. $\mathrm{Q}$ ranges from a value of 0 at $\mathrm{t}_{0}$ to 1.0 after Triton addition.

\section{Abbreviations}

Ab: antibody; BCL: bovine cardiolipin; CB: Cascade Blue; DDM: n-dodecyl- $\beta$ D-maltoside; FL: fluorescence; HA: hyaluronic acid: hyaluronate: hyaluronan; HAS: HA synthase; MD: membrane domain; MRP5: multidrug resistant protein 5; NEM: N-ethylmaleimide; PBS: phosphate buffered saline; PE: phospatidyl ethanolamine; PG: phosphatidyl glycerol; PL: phospholipid; SeHAS: Streptococcus equisimilis HAS; SpHAS: Streptococcus pyogenes HAS; TM-CL: tetramyristoyl cardiolipin TO-CL: tetraoleoyl cardiolipin.

\section{Acknowledgements}

We thank Amy Padgett-McCue and Jennifer Washburn for technical assistance and Jun Peng and Feng He for technical advice. This research was supported by National Institute of General Medical Sciences grant GM35978 (PHW) and GM062964 (JL) from the National Institutes of Health. 


\section{Author details}

'Department of Biochemistry \& Molecular Biology, The University of Oklahoma Health Sciences Center (940 S. L. Young Blvd), Oklahoma City, OK 73104, USA. ${ }^{2}$ The Peggy and Charles Stephenson Oklahoma Cancer Center, The University of Oklahoma Health Sciences Center (940 S. L. Young Blvd), Oklahoma City, OK 73104, USA. ${ }^{3}$ The Oklahoma Center for Medical Glycobiology, The University of Oklahoma Health Sciences Center (940 S. L. Young Blvd), Oklahoma City, OK 73104, USA.

\section{Authors' contributions}

APM performed all the experiments and helped interpret results and draft the manuscript. JL served as an expert consultant regarding liposomes, helped plan liposome-based studies, provided instrument and software training and helped draft the manuscript. PHW conceived of and planned the approach, and helped design experiments, interpret results, and draft the manuscript. All authors have read and approved the final manuscript.

Received: 15 November 2011 Accepted: 25 January 2012 Published: 25 January 2012

\section{References}

1. Weigel PH, DeAngelis PL: Hyaluronan synthases: A decade-plus of novel glycosyltransferases. J Biol Chem 2007, 282:36777-36781.

2. Heldermon C, DeAngelis PL, Weigel PH: Topological organization of the hyaluronan synthase from Streptococcus pyogenes. J Biol Chem 2001, 276:2037-2046.

3. McDonald J, Hascall VC: Hyaluronan minireview series. J Biol Chem 2002, 277:4575-4579.

4. Stern $R$, Asari AA, Sugahara KN: Hyaluronan fragments: an informationrich system. Eur J Cell Biol 2006, 85:699-715.

5. Noble PW: Hyaluronan and its catabolic products in tissue injury and repair. Matrix Biol 2002, 21:25-29.

6. Noble PW, McKee CM, Cowman M, Shin HS: Hyaluronan fragments activate an NF-kappa B/I-kappa B alpha autoregulatory loop in murine macrophages. J Exp Med 1996, 183:2373-2378.

7. Pardue EL, Ibrahim S, Ramamurthi A: Role of hyaluronan in angiogenesis and its utility to angiogenic tissue engineering. Organogenesis 2008 , 4:203-214.

8. West DC, Kumar S: Hyaluronan and angiogenesis. Ciba Found Symp 1989, 143:187-201.

9. Tlapak-Simmons VL, Baggenstoss BA, Clyne T, Weigel PH: Purification and lipid dependence of the recombinant hyaluronan synthases from Streptococcus pyogenes and Streptococcus equisimilis. J Biol Chem 1999, 274:4239-4245.

10. Tlapak-Simmons VL, Kempner ES, Baggenstoss BA, Weigel PH: The active streptococcal hyaluronan synthases (HASs) contain a single HAS monomer and multiple cardiolipin molecules. J Biol Chem 1998, 273:26100-26109.

11. Ouskova G, Spellerberg B, Prehm P: Hyaluronan release from Streptococcus pyogenes: export by an $A B C$ transporter. Glycobiology 2004, 14:931-938.

12. Prehm P, Schumacher U: Inhibition of hyaluronan export from human fibroblasts by inhibitors of multidrug resistance transporters. Biochem Pharmacol 2004, 68:1401-1410.

13. Schulz T, Schumacher U, Prante C, Sextro W, Prehm P: Cystic fibrosis transmembrane conductance regulator can export hyaluronan. Pathobiology 2010, 77:200-209.

14. Schulz T, Schumacher U, Prehm P: Hyaluronan export by the ABC transporter MRP5 and its modulation by intracellular CGMP. I Biol Chem 2007, 282:20999-21004

15. Baggenstoss BA, Weigel PH: Size exclusion chromatography-multiangle laser light scattering analysis of hyaluronan size distributions made by membrane-bound hyaluronan synthase. Anal Biochem 2006, 352:243-251

16. Widner B, Behr R, Von Dollen S, Tang M, Heu T, Sloma A, et al: Hyaluronic acid production in Bacillus subtilis. Appl Environ Microbiol 2005, 71:3747-3752

17. DeAngelis PL, Papaconstantinou J, Weigel PH: Molecular cloning, identification and sequence of the hyaluronan synthase gene from group A streptococcus pyogenes. J Biol Chem 1993, 268:19181-19184.
18. Takeo S, Fujise M, Akiyama T, Habuchi H, Itano N, Matsuo T, et al: In vivo hyaluronan synthesis upon expression of the mammalian hyaluronan synthase gene in drosophila. J Biol Chem 2004, 279:18920-18925.

19. Peng J, Tan C, Roberts GJ, Nikolaeva O, Zhang Z, Lapolla SM, et al: tBid elicits a conformational alteration in membrane-bound $\mathrm{BCl}-2$ such that it inhibits Bax pore formation. J Biol Chem 2006, 281:35802-35811.

20. Peng J, Ding J, Tan C, Baggenstoss B, Zhang Z, Lapolla SM, et al: Oligomerization of membrane-bound $\mathrm{BCl}-2$ is involved in its pore formation induced by tBid. Apoptosis 2009, 14:1145-1153.

21. Tlapak-Simmons VL, Baron CA, Weigel PH: Characterization of the purified hyaluronan synthase from Streptococcus equisimilis. Biochem 2004, 43:9234-9242.

22. Weigel PH, Kyossev Z, Torres LC: Phospholipid dependence and liposome reconstitution of purified hyaluronan synthase. J Biol Chem 2006, 281:36542-36551.

23. Dorfman A, Markovitz A, Cifonelli JA: Metabolism of hyaluronic acid and chondroitinsulfuric acids. Fed Proc 1958, 17:1093-1099.

24. Kumari K, Tlapak-Simmons VL, Baggenstoss BA, Weigel PH: The streptococcal hyaluronan synthases are inhibited by sulfhydryl modifying reagents but conserved cysteine residues are not essential for enzyme function. J Biol Chem 2002, 277:13943-13951.

25. Heldermon CD, Tlapak-Simmons VL, Baggenstoss BA, Weigel PH: Sitedirected mutation of conserved cysteine residues does not inactivate the Streptococcus pyogenes hyaluronan synthase. Glycobiology 2001, 11:1017-1024

26. Kumari K, Weigel PH: Identification of a membrane-localized cysteine cluster near the substrate binding sites of the streptococcus equisimilis hyaluronan synthase. Glycobiology 2005, 15:529-539.

27. Kumari K, Baggenstoss BA, Parker AL, Weigel PH: Mutation of two intramembrane polar residues conserved within the hyaluronan synthase family alters hyaluronan product size. J Biol Chem 2006, 281:11755-11760.

28. Renner LD, Weibel DB: Cardiolipin microdomains localize to negatively curved regions of Escherichia coli membranes. Proc Natl Acad Sci USA 2011, 108:6264-6269.

29. Huang KC, Ramamurthi KS: Macromolecules that prefer their membranes curvy. Mol Microbiol 2010, 76:822-832.

30. DeAngelis PL, Papaconstantinou J, Weigel PH: Isolation of a Streptococcus pyogenes gene locus that directs hyaluronan biosynthesis in acapsular mutants and in heterologous bacteria. J Biol Chem 1993, 268:14568-14571.

31. Weigel PH: The bacterial hyaluronan synthases - an update. In Science of Hyaluronan Today Edited by: Hascall VC, Yanagishita M 2004 [http://www. glycoforum.gr.jp/science/hyaluronan/HA06a/HA06aE.html], Ref Type: Electronic Citation.

32. Lundbaek JA, Collingwood SA, Ingolfsson HI, Kapoor R, Andersen OS: Lipid bilayer regulation of membrane protein function: gramicidin channels as molecular force probes. J R Soc Interface 2010, 7:373-395.

33. Lundbaek JA: Lipid bilayer-mediated regulation of ion channel function by amphiphilic drugs. J Gen Physiol 2008, 131:421-429.

34. Lundbaek JA, Maer AM, Andersen OS: Lipid bilayer electrostatic energy, curvature stress, and assembly of gramicidin channels. Biochemistry 1997, 36:5695-5701.

35. Tan C, Dlugosz PJ, Peng J, Zhang Z, Lapolla SM, Plafker SM, et al: Autoactivation of the apoptosis protein Bax increases mitochondrial membrane permeability and is inhibited by Bcl-2.J Biol Chem 2006, 281:14764-14775.

36. DeAngelis PL, Weigel PH: Immunochemical confirmation of the primary structure of streptococcal hyaluronan synthase and synthesis of high molecular weight product by the recombinant enzyme. Biochem 1994, 33:9033-9039.

37. Pratt S, Shepard RL, Kandasamy RA, Johnston PA, Perry W, Dantzig AH: The multidrug resistance protein 5 ( $A B C C 5$ ) confers resistance to 5 fluorouracil and transports its monophosphorylated metabolites. $\mathrm{Mol}$ Cancer Ther 2005, 4:855-863.

38. Thomas NK, Brown TJ: ABC transporters do not contribute to extracellular translocation of hyaluronan in human breast cancer in vitro. Exp Cell Res 2010.

39. Szoka F, Olson F, Heath T, Vail W, Mayhew E, Papahadjopoulos D: Preparation of unilamellar liposomes of intermediate size (0.1-0.2 $\mu \mathrm{m})$ 
by a combination of reverse phase evaporation and extrusion through polycarbonate membranes. Biochim Biophys Acta 1980, 601:559-571.

40. Zhang Z, Lapolla SM, Annis MG, Truscott M, Roberts GJ, Miao Y, et al: Bcl-2 homodimerization involves two distinct binding surfaces, a topographic arrangement that provides an effective mechanism for $\mathrm{Bcl}-2$ to capture activated Bax. J Biol Chem 2004, 279:43920-43928.

doi:10.1186/1471-2091-13-2

Cite this article as: Medina et al:: Hyaluronan synthase mediates dye

translocation across liposomal membranes. BMC Biochemistry 2012 13:2.

Submit your next manuscript to BioMed Central and take full advantage of:

- Convenient online submission

- Thorough peer review

- No space constraints or color figure charges

- Immediate publication on acceptance

- Inclusion in PubMed, CAS, Scopus and Google Scholar

- Research which is freely available for redistribution

Submit your manuscript at www.biomedcentral.com/submit 